\title{
Preoperative serum CA-125 level as a predictor for the extent of cytoreduction in patients with advanced stage epithelial ovarian cancer
}

\author{
Sebastjan Merlo ${ }^{1,2}$, Nikola Besic ${ }^{2,3}$, Eva Drmota ${ }^{2}$, Nina Kovacevic ${ }^{1,2,4}$ \\ 1 Department of Gynaecological Oncology, Institute of Oncology Ljubljana, Ljubljana, Slovenia \\ ${ }^{2}$ Faculty of Medicine, University of Ljubljana, Ljubljana, Slovenia \\ ${ }^{3}$ Department of Surgical Oncology, Institute of Oncology Ljubljana, Ljubljana, Slovenia \\ ${ }^{4}$ Faculty of Health Care Angele Boškin, Jesenice, Slovenia
}

Radiol Oncol 2021; 55(3): 341-346.

Received 25 November 2020

Accepted 9 February 2021

Correspondence to: Assist. Nina Kovačević M.D., Ph.D., Department of Gynaecological Oncology, Institute of Oncology Ljubljana, Zaloška cesta 2, SI-1000 Ljubljana, Slovenia. Phone: + 38641277 602; E-mail: nkovacevic@onko-i.si

Disclosure: No potential conflicts of interest were disclosed.

This is an open access article under the CC BY-NC-ND license (http://creativecommons.org/licenses/by-nc-nd/4.0/).

Background. Ovarian cancer is the seventh most common cancer in women worldwide and the eighth most common cause of cancer death. Due to the lack of effective early detection strategies and the unspecific onset of symptoms, it is diagnosed at an advanced stage in $75 \%$ of cases. The cancer antigen (CA) 125 is used as a prognostic marker and its level is elevated in more than $85 \%$ of women with advanced stages of epithelial ovarian cancer (EOC). The standard treatment is primary debulking surgery (PDS) followed by adjuvant chemotherapy (ACT), but the later approach is neoadjuvant chemotherapy (NACT) followed by interval debulking surgery (IDS). Several studies have been conducted to find out whether preoperative CA-125 serum levels influence treatment choice, surgical resection and survival outcome. The aim of our study was to analyse experience of single institution as Cancer comprehensive center with preoperative usefulness of CA-125.

Patients and methods. At the Institute of Oncology Ljubljana a retrospective analysis of 253 women with stage FIGO IIIC and IV ovarian cancer was conducted. Women were divided into two groups based on their primary treatment. The first group was the NACT group ( 215 women) and the second the PDS group (38 women). The differences in patient characteristics were compared using the Chi-square test and ANOVA and the Kaplan-Meier method was used for calculating progression-free survival (PFS) and overall survival (OS).

Results. The median serum CA-125 level was higher in the NACT group than in the PDS group, $972 \mathrm{IU} / \mathrm{ml}$ and $499 \mathrm{IU} /$ $\mathrm{ml}$, respectively. The PFS in the NACT group was 8 months $(95 \% \mathrm{Cl} 6.4-9.5)$ and 18 months $(95 \% \mathrm{Cl} 12.5-23.4)$ in the PDS group. The median OS was lower in the NACT group than in the PDS group, 25 months (95\% Cl 20.6-29.5) and 46 months (95\% Cl 32.9-62.1), respectively.

Conclusions. Preoperative CA-125 cut off value of $500 \mathrm{IU} / \mathrm{ml}$ is a promising threshold to predict a successful PDS.

Key words: ovarian cancer; tumour marker; CA-125; primary debulking surgery; neoadjuvant chemotherapy

\section{Introduction}

Ovarian cancer is the seventh most common cancer in women around the world, with approximately 240,000 new cases diagnosed each year. Epithelial ovarian cancer $(E O C)$ is a very aggressive disease and is the eighth leading cause of cancer death with five-year survival rates below $45 \% .^{1}$ Of all patients diagnosed with EOC, approximately $15 \%$ of patients will have germline BRCA1 or BRCA2 mutation present. The cumulative ovarian cancer risk to age of 80 is $36-53 \%$ in BRCA1 mutation and $11-25 \%$ in BRCA2 mutation. Cumulative ovarian risk to age of 80 in population without BRCA mutation is $1-2 \%{ }^{2,3}$ 
Due to the lack of effective screening strategies and the unspecific onset of symptoms, EOC is detected in $75 \%$ of cases at an advanced stage. ${ }^{4}$ The initial symptoms are persistent or frequent, nonspecific and mainly include abdominal distension or flatulence, pelvic or abdominal pain, bloating, loss of appetite, unexplained weight loss, fatigue or changes in bowel habits. About $36 \%$ of women with unspecific clinical symptoms make several visits to their general practitioner before being diagnosed with ovarian cancer. ${ }^{5}$

CA-125, also known as mucin 16 or MUC16, is a large membrane glycoprotein belonging to the wide mucin family, encoded by the homonymous MUC16 gene. ${ }^{6}$ It can be very useful and highly specific as a prognostic maker, but not as a diagnostic tool due to lack of sensitivity. ${ }^{7}$ CA-125 level is increased in more than $85 \%$ of women with an advanced stage EOC but is only increased in 50\% of stage I cancers. ${ }^{7,8}$ CA-125 level may also be increased in almost $6 \%$ of women without ovarian cancer due to adenomyosis, endometriosis, retrograde menstrual bleeding or other non-malignant diseases. $^{7}$

For decades the standard treatment for EOC has been primary debulking surgery (PDS) followed by platinum- and taxane-based adjuvant chemotherapy (ACT). A more contemporary approach is neoadjuvant chemotherapy (NACT) followed by interval debulking surgery (IDS), but, opinions on the optimal treatment are still divided. ${ }^{9}$ Despite different treatment approaches, the prognosis is mainly influenced by the residual disease after surgical cytoreduction. Patients benefit most from complete gross resection or optimal cytoreduction (residual lesions with a diameter of $1 \mathrm{~cm}$ or less). Suboptimal cytoreduction with residual disease of more than $1 \mathrm{~cm}$ is associated with poorer survival. ${ }^{10}$ Many studies have attempted to assess different scoring systems which include preoperative serum CA-125 to determine the patient selection where optimal primary cytoreduction might be achievable but results and recommendations are inconsistent. ${ }^{11-16}$

The aim of our study was to analyse experience of single institution as Cancer comprehensive centre with preoperative usefulness of CA-125.

\section{Patients and methods}

\section{Study design}

A retrospective observational study was conducted at the Institute of Oncology Ljubljana, from January
2005 to December 2014. The data collection and its analysis were approved by Institutional Ethical committee.

\section{Patients}

Two hundred and fifty-three women with advanced stage ovarian cancer were enrolled in the study. All women had histologically confirmed FIGO stage IIIC and IV EOC. Women were divided into two groups based on their initial treatment. The first group consisted of 215 women receiving NACT (based on carboplatin and paclitaxel), followed by IDS. The second group consisted of 38 women treated with PDS, followed by ACT (3 courses of the same regimen as NACT). The selection of women for the specific treatment was based on the ability to perform a complete gross resection or to achieve a residual disease of $1 \mathrm{~cm}$ or less. This decision was based on preoperative imaging studies (abdominal and thoracic CT) and/or diagnostic laparoscopy in 173 patients. Levels of preoperative CA-125 did not influence the decision about primary treatment modality. When option to achieve complete gross or at least optimal cytoreduction was considered low, NACT was selected. Patients were assessed as inoperable (low probability of $<1 \mathrm{~cm}$ residual disease) if the tumour penetrated the pelvic wall, if carcinosis of the intestine, intestinal serosa or mesentery was present or if imaging studies showed tumour spread to distant organs. The time from NACT to IDS was 4-6 weeks and the interval from PDS to ACT was 3-4 weeks for all included women.

Progression-free survival (PFS) was defined as the time from the date of completion of treatment to the first radiological evidence of progression. An increase of CA-125 serum level without clinical signs of recurrence was not counted as progression, but triggered further radiological examinations. Overall survival (OS) was defined as the interval between the date of diagnosis and the date of death. The surviving patients were censored at the time of the last follow-up.

The extent of residual disease was based on the diameter of the largest single lesion. At complete gross resection there were no macroscopic lesions, at optimal resection the lesions had a diameter of $1 \mathrm{~cm}$ or less and at suboptimal resection the lesions were larger than $1 \mathrm{~cm}$. The study excluded women with a history of other malignant tumours or chemotherapy, FIGO stage I or II ovarian cancer, or non-epithelial histology of ovarian cancer. 


\section{Data collection}

Patients enrolled in the study were selected using the prospective clinical database of the Institute of Oncology Ljubljana. Clinical variables were collected from electronic hospital records, paper documentation and pathology reports to determine eligibility for the study, general characteristics of the patients, FIGO stage, tumour classification and histological type. Vital status was determined by analysing electronic medical records. Data were collected on the patient's age, body mass index (BMI), menopause status, preoperative CA-125, duration of follow-up and residual disease after surgery.

\section{Statistical analysis}

For demographic data, descriptive statistics were used. The median survival of the two groups was calculated based on the non-normal distribution. The differences in patient characteristics were compared using the Chi-square test and ANOVA. ROC analysis was performed to determine cuff off values of serum CA-125 levels. PFS and OS and were estimated using the Kaplan-Meier method, and the rates in the two groups were compared using the log-rank test. $\mathrm{P}<0.05$ indicated that the difference between the groups was statistically significant. The statistical software SPSS for Windows version 26 was used for statistical analysis.

\section{Results}

A retrospective analysis of 253 patients with advanced stage EOC treated at the Institute of Oncology Ljubljana between January 2005 and December 2014 was performed. There were 215 $(84.9 \%)$ women enrolled in the NACT group and $38(15.1 \%)$ women in the PDS group. The characteristics of the patients are shown in Table 1. Patients in the PDS group were statistically significantly younger (53.7 vs. 62.2 years), with lower disease stage (FIGO IIIC $89.5 \%$ vs. $66.6 \%$ ) and had lower CA-125 levels (499 IU/ml vs. $972 \mathrm{IU} / \mathrm{ml}$ ).

In patients with NACT, 57.6\% (124/215) had complete gross resection, $14.0 \%(30 / 215)$ had optimal resection and $28.4 \%(61 / 215)$ had suboptimal resection $(\mathrm{p}=0.002$; Table 1$)$.

In women with PDS, 23.7\% (9/38) had complete gross resection, $18.4 \%(7 / 38)$ had optimal resection and $57.9 \%(22 / 38)$ had suboptimal resection. Patients with complete gross resection had low-
TABLE 1. Clinical characteristics $(N=253)$

\begin{tabular}{|c|c|c|c|}
\hline Characteristic & $\begin{array}{c}\text { PDS } \\
(\mathrm{N}=38)\end{array}$ & $\begin{array}{c}\text { NACT } \\
(\mathrm{N}=215)\end{array}$ & $P$ value \\
\hline \multicolumn{4}{|l|}{ Age-years } \\
\hline Median & 53.7 & 62.2 & $<0.001$ \\
\hline Range & $29-84$ & $39-85$ & \\
\hline \multicolumn{4}{|l|}{ BMI-kg/m² } \\
\hline Median & 24.5 & 23.8 & 0.210 \\
\hline Range & $17.4-45.2$ & $18.2-32.1$ & \\
\hline \multicolumn{4}{|l|}{ Parity-number } \\
\hline Median & 2 & 2 & 0.080 \\
\hline Range & $0-5$ & $0-4$ & \\
\hline \multicolumn{4}{|l|}{ Menopause-years } \\
\hline Median & 50 & 51.5 & 0.340 \\
\hline Range & $37-60$ & $45-58$ & \\
\hline \multicolumn{4}{|l|}{ ASA score } \\
\hline 1 & $7(18.4)$ & $23(10.7)$ & \multirow{4}{*}{0.780} \\
\hline 2 & $22(57.9)$ & $141(65.6)$ & \\
\hline 3 & $9(23.7)$ & 49 (22.8) & \\
\hline 4 & $0(0)$ & $2(0.9)$ & \\
\hline \multicolumn{4}{|l|}{ WHO performance status } \\
\hline 0 & $26(68.4)$ & $96(44.7)$ & \multirow{5}{*}{0.130} \\
\hline 1 & $8(21.0)$ & $85(40.0)$ & \\
\hline 2 & $4(10.6)$ & $26(12.1)$ & \\
\hline 3 & $0(0)$ & $5(2.3)$ & \\
\hline 4 & $0(0)$ & $3(1.4)$ & \\
\hline \multicolumn{4}{|l|}{ FIGO stage } \\
\hline IIIC & $34(89.5)$ & $143(66.6)$ & \multirow{2}{*}{0.010} \\
\hline IV & $4(10.5)$ & $72(33.4)$ & \\
\hline \multicolumn{4}{|l|}{ Histologic type } \\
\hline Serous & $32(84.2)$ & $202(94.0)$ & \multirow{4}{*}{0.100} \\
\hline Endometrioid & $6(15.8)$ & $8(3.7)$ & \\
\hline Mucinous & $0(0)$ & $3(1.4)$ & \\
\hline Clear-cell & $0(0)$ & $2(0.9)$ & \\
\hline \multicolumn{4}{|l|}{ Preoperative CA-125- IU/ml } \\
\hline Median & 499 & 972 & 0.058 \\
\hline Range & $59-5739$ & $10-31481$ & \\
\hline \multicolumn{4}{|l|}{ Surgical outcome } \\
\hline Complete gross resection & $9(23.7)$ & $124(57.6)$ & \multirow{3}{*}{0.002} \\
\hline Optimal visible residual $(\leq 1 \mathrm{~cm})$ & $7(18.4)$ & $30(14.0)$ & \\
\hline Suboptimal $(>1 \mathrm{~cm})$ & $22(57.9)$ & $61(28.4)$ & \\
\hline \multicolumn{4}{|l|}{ Hospitalisation time-days } \\
\hline Median & 10 & 9 & 0.555 \\
\hline Range & $7-28$ & $5-59$ & \\
\hline
\end{tabular}

$\mathrm{ASA}=$ American Society of Anesthesiologist; $\mathrm{BMI}=$ body mass index; $\mathrm{FIGO}=$ International Federation of Gynecology and Obstetrics; NACT = neoadjuvant chemotherapy; PDS = primary debulking surgery; $\mathrm{WHO}=$ World Health Organization 
TABLE 2. Median and range CA-125 levels in different surgical outcomes in primary debulking surgery (PDS) and = neoadjuvant chemotherapy (NACT) group

\begin{tabular}{|cccc}
\hline Surgical outcomes & PDS $(\mathbf{N}=\mathbf{3 8})$ & NACT $(\mathbf{N}=\mathbf{2 1 5})$ & P value \\
\hline CA-125 at diagnosis IU/ml & & & \\
\hline Complete gross resection & $559-5739$ & $10-12803$ & \\
\hline Optimal resection & 512 & 1006 & 0.006 \\
\hline Suboptimal resection & $85-1117$ & $48-24824$ & \\
\hline CA-125 post NACT IU/ml & 1522 & 1063 & \\
\hline Complete gross resection & $200-3569$ & $28-31481$ & \\
\hline Optimal resection & & 25 & 0.020 \\
\hline Suboptimal resection & & $5-2074$ & \\
\hline
\end{tabular}

CA-125 = cancer antigen 125

est CA-125 level at the time of diagnosis, $359 \mathrm{IU} /$ $\mathrm{ml}$ respectively. Highest CA-125 level was found in the group with suboptimal resection, 1522 IU/ $\mathrm{ml}$, respectively.

CA-125 level in NACT group with complete gross resection at the time of diagnosis was $943 \mathrm{IU} /$ $\mathrm{mL}$ and after NACT $25 \mathrm{IU} / \mathrm{ml}$ (97.3\% decline). CA125 level in NACT group with optimal resection at the time of diagnosis was $1006 \mathrm{IU} / \mathrm{ml}$ and after NACT $36 \mathrm{IU} / \mathrm{ml}$ (96.4\% decline). Serum CA-125 level in NACT group with suboptimal resection at the time of diagnosis was $1063 \mathrm{IU} / \mathrm{ml}$ and after NACT $68 \mathrm{IU} / \mathrm{ml}$ (93.6\% decline) (Table 2).

Cut off values of serum CA-125 levels and sensitivity to obtain complete gross or optimal cytoreduction are shown in Table 3. If CA-125 preoperative serum level is $250 \mathrm{IU} / \mathrm{ml}$, there is $74 \%$ chance to obtain at least optimal cyoreduction (Table 3 ).

PFS in the NACT group was 8 months $(95 \% \mathrm{CI}$ : 6.4-9.5) and 18 months (95\% CI: 12.5-23.4) in the PDS group ( $\mathrm{P}=0.008)$.

The median OS in the NACT group was 25 months (95\% CI: 20.6-29.5) and 46 months (95\% CI: $32.9-62.1)$ in the PDS group $(p=0.009)$.

\section{Discussion}

PDS followed by platinum- and taxane-based ACT was the standard treatment for patients with advanced stage EOC until 2016, when the American Society of Clinical Oncology (ASCO) and the Society of Gynecologic Oncology (SGO) developed new clinical practice guidelines. ${ }^{17}$
TABLE 3. Statistical cut off values of serum CA-125 level and probability to obtain complete gross or optimal cytoreduction

\begin{tabular}{cccc}
\hline $\begin{array}{c}\text { CA-125 level } \\
(\text { IU/ml) }\end{array}$ & $\begin{array}{c}\text { Sensitivity } \\
\text { (\%) }\end{array}$ & $\begin{array}{c}\text { Number and percentage } \\
\text { of patients }\end{array}$ \\
\hline 50 & 96.5 & 9 & $3.5 \%$ \\
100 & 86.5 & 33 & $13.0 \%$ \\
250 & 74.0 & 66 & $26.1 \%$ \\
500 & 58.0 & 98 & $38.7 \%$ \\
750 & 50.0 & 128 & $50.6 \%$ \\
1000 & 42.0 & 150 & $59.3 \%$ \\
\hline
\end{tabular}

CA-125 = cancer antigen 125

According to these guidelines in patients with high likelihood of achieving cytoreduction with residual disease $<1 \mathrm{~cm}$ (ideally no visible disease) with acceptable morbidity, PDS is recommended over NACT. For women with high perioperative risk or a low likelihood of achieving a cytoreduction with residual disease $<1 \mathrm{~cm}$ (ideally no visible disease) NACT is the treatment of choice. For women who are fit for PDS but cytoreduction with residual disease $<1 \mathrm{~cm}$ (ideally no visible disease) is unlikely, NACT is also the treatment of choice. IDS should be performed after three to six cycles of NACT for women who respond to chemotherapy or with stable disease. ${ }^{9,17}$ Patients with disease progression during NACT have a poor prognosis. Options include alternative chemotherapy regimens, inclusion in clinical trials and/or discontinuation of active cancer therapy and initiation of palliative supportive care. The role of surgery in palliative care is limited. ${ }^{18,19}$

There were studies published before year 2016 which showed non-inferiority of NACT compared to PDS. ${ }^{20-23}$ Therefore also at our institute patients were treated with NACT where low chances to achieve at least optimal PDS were expected.

We studied the use of preoperative serum CA125 levels to predict the likelihood of achieving at least optimal PDS or IDS in patients with advanced stage EOC.

Many studies have attempted to assess the ability of preoperative serum CA-125 level and various scoring systems to determine the patient selection where optimal primary cytoreduction can be achieved.

As expected, our results confirmed that the higher the CA-125 level is, the lower is probability to achieve optimal cytoreduction. At the preoperative CA-125 cut off value of $500 \mathrm{IU} / \mathrm{ml}$ the probability of achieving complete gross or at least 
optimal cytoreduction in patients with advanced stage EOC was $58 \%$. The probability increased to $74 \%$ at the cut off value of $250 \mathrm{IU} / \mathrm{ml}$. These results add further data on usefulness of CA-125 levels as predictive factor for type of resection and are consistent with results published by other authors. ${ }^{12,13}$

Vorgias et al. ${ }^{12}$ and Kang et al. ${ }^{13}$ showed that CA-125 level above $500 \mathrm{IU} / \mathrm{ml}$ correlates with a more complex and radical surgical procedure and a worse outcome. Cut off value of $500 \mathrm{IU} / \mathrm{ml}$ had sensitivity ranging from $49 \%$ to $78 \%$ and specificity ranging from $59 \%$ to $77 \%$. They reported that CA-125 levels above $500 \mathrm{IU} / \mathrm{ml}$ were strongly correlated with a suboptimal cytoreduction and poorer overall survival in patients with advanced EOC.

Furthermore, Arab et al. ${ }^{11}$ established a model for predicting optimal surgical outcome, in which a CA125 value of $420 \mathrm{IU} / \mathrm{ml}$ or less, the absence of massive ascites and liver metastases were shown to be significant factors in achieving optimal cytoreduction.

However, Chi et al..$^{14}$ reported that preoperative CA-125 value of more than $500 \mathrm{IU} / \mathrm{ml}$ had no predictive usefulness on the surgical outcome after an extensive upper abdominal surgery. A preoperative value of CA- 125 above $500 \mathrm{IU} / \mathrm{ml}$ was associated with a probability of only $22 \%$ for optimal cytoreduction, but when extensive upper abdominal surgery was performed, the rate of optimal cytoreduction increased to $75 \%$ and the preoperative CA125 value was no longer an independent predictor of surgical outcome. ${ }^{14}$

If optimal PDS is not achievable or patients are not suitable for extensive surgery, NACT and IDS are indicated. These patients usually have higher preoperative CA-125 levels and a higher disease burden than patients treated with a PDS. This was also the case in our patients where patients with NACT and IDS had higher disease stage (FIGO IV $33.4 \%$ vs. $10.5 \%$ ) and higher preoperative CA-125 levels (972 IU/ml vs. $499 \mathrm{IU} / \mathrm{ml})$.

Rodriguez et al. ${ }^{15}$ reported that a preoperative CA-125 level of less than $100 \mathrm{IU} / \mathrm{ml}$ may be a suitable predictor of complete gross resection rather than optimal cytoreduction. According to our results preoperative CA-125 levels of less than 100 $\mathrm{IU} / \mathrm{ml}$ can be expected in only $13 \%$ of patients. At an arbitrary cut off value of $250 \mathrm{IU} / \mathrm{ml}$ the probability to obtain complete gross or optimal cytoreduction is $75 \%$ and roughly one forth $(26.1 \%)$ of all patients with EOC belong to this group. If we increase the cut off value to $500 \mathrm{IU} / \mathrm{ml}$ about one third $(38.7 \%)$ of patients will be included and the probability to obtain complete gross or optimal cytoreduction will be $58 \%$.
After NACT the role of CA-125 to predict complete gross or optimal cytoreduction is even more complex. Pelissier et al. ${ }^{16}$ found out that a preoperative CA-125 level of less than $75 \mathrm{IU} / \mathrm{ml}$ after the third cycle of NACT predicted a complete IDS. CA125 of less than $200 \mathrm{IU} / \mathrm{ml}$ can be an independent predictor of complete gross IDS and also a predictor of chemosensitivity according to Zeng et al. ${ }^{24}$. However, after NACT the percentage of reduction is probably even more important than the absolute decrease in the CA-125 value. A reduction of at least $90 \%$ indicates a better response of the tumour to treatment and therefore correlates with a better surgical outcome and better overall survival. ${ }^{25}$

Our study showed that patients with CA-125 serum reduction of more than $96.4 \%$ achieved higher complete gross and optimal IDS rate in comparison to patients with lover reduction of serum CA-125 level. It is well established that CA-125 serum level represents the tumour burden in most patients with advanced stage EOC. Sharp CA-125 serum level reduction during NACT might reflect the chemosensitivity of the tumour. This might be a predictive factor for surgical outcome. However, we cannot predict in advance the reduction of CA125 level and therefore cannot predict the benefit of NACT.

A study published by Gupta et al. ${ }^{26}$ showed that a 95\% reduction of CA-125 levels and an absolute preoperative CA-125 level of $100 \mathrm{IU} / \mathrm{ml}$ or less predicted complete gross resection after NACT.

Kessous et al. ${ }^{27}$ think that the regression coefficient is impractical for clinical daily routine and found out that an early reduction of CA-125 levels by the third cycle of NACT can best predict surgical outcome and patients overall survival.

Our study showed that NACT group had shorter OS and PSF compare to PDS group. The median PFS and OS for patients in the NACT group was 8 and 25 months, compared to 25 and 49 months in the PDS group, respectively. This is consistent with data published by Mueller et al..$^{28}$ but in contrast to EORTC/NCIC and CHORUS study. ${ }^{20,29}$ The explanation for our results is that women treated with NACT had a higher disease burden, a higher FIGO stage, a higher CA-125 level at time of diagnosis and were older in comparison to PDS group.

Patients included in before mentioned three studies were of comparable age, FIGO stage and had comparable preoperative CA-125 levels.

According to Maner and Machida NACT can be associated with lower peri- and postoperative morbidity and mortality and shorter hospital stay but PDS may offer a better chance of survival in 
selected patients. ${ }^{30,31}$ Median hospital stay in our patients treated with IDS (9 days) was not different from patients treated with PDS (10 days, $\mathrm{p}=$ $0.555)$ which is consistent with the study published by du Bois. ${ }^{32}$ However, patients treated with PDS had better survival as already mentioned.

There are limitations to our study among which are its retrospective nature and that there were no generally accepted selection criteria at that time for which patients are candidates for PDS or NACT.

\section{Conclusions}

Preoperative CA-125 cut off value of $500 \mathrm{IU} / \mathrm{ml}$ is a promising threshold to predict a successful PDS. After NACT a decline of CA-125 of more than $96.4 \%$ predicts at least optimal cytoreduction of IDS.

\section{References}

1. Momenimovahed Z, Tiznobaik A, Taheri S, Salehiniya H. Ovarian cancer in the world: epidemiology and risk factors. Int J Womens Health 2019; 11 287-99. doi: 10.2147/IJWH.S197604

2. Kuchenbaecker KB, Hopper JL, Barnes DR, Phillips K-A, Mooij TM, RoosBlom $\mathrm{MJ}$, et al. Risks of breast, ovarian, and contralateral breast cancer for BRCA1 and BRCA2 mutation carriers. JAMA 2017; 317: 2402-16. doi: 10.1001/jama.2017.7112

3. Neff RT, Senter L, Salani R. BRCA mutation in ovarian cancer: testing, implications and treatment considerations. Ther Adv Med Oncol 2017; 9: 519-31. doi: 10.1200/JCO.2003.01.068

4. Bast RC Jr. Status of tumor markers in ovarian cancer screening. J Clin Oncol 2003; 21(10 Suppl): 200s-5s. doi: 10.1200/JCO.2003.01.068

5. Yawn BP, Barrette BA, Wollan PC. Ovarian cancer: the neglected diagnosis. Mayo Clin Proc 2004; 79: 1277-82. doi: 10.4065/79.10.1277

6. Bottoni P, Scatena R. The role of CA 125 as tumor marker: biochemical and clinical aspects. Adv Exp Med Biol 2015; 867: 229-44. doi: 10.1007/978-94 017-7215-0_14

7. Yang $\mathrm{WL}, \mathrm{Lu} Z$, Bast RC. The role of biomarkers in the management of epithelial ovarian cancer. Expert Rev Mol Diagn 2017; 17: 577-91. doi: 10.1080/14737159.2017

8. Sundar S, Neal RD, Kehoe S. Diagnosis of ovarian cancer. BMJ 2015; 351: h4443. doi: https://doi.org/10.1136/bmj.h4443

9. Elies A, Rivière S, Pouget N, Becette V, Dubot C, Donnadieu A, et al. The role of neoadjuvant chemotherapy in ovarian cancer. Expert Rev Anticancer Ther 2018; 18: 555-66. doi: 10.1080/14737140.2018.1458614

10. du Bois A, Reuss A, Pujade-Lauraine E, Harter P, Ray-Coquard I, Pfisterer J. Role of surgical outcome as prognostic factor in advanced epithelial ovarian cancer: a combined exploratory analysis of 3 prospectively randomized phase 3 multicenter trials: by the Arbeitsgemeinschaft Gynaekologische Onkologie Studiengruppe Ovarialkarzinom (AGO-OVAR) and the Groupe d'Investigateurs Nationaux Pour les Etudes des Cancers de l'Ovaire (GINECO). Cancer 2009; 115: 1234-44. doi: 10.1002/cncr.24149

11. Arab M, Jamdar F, Sadat Hosseini M, Ghodssi-Ghasemabadi R, Farzaneh $F$, Ashrafganjoie T. Model for prediction of optimal debulking of epithelial ovarian cancer. Asian Pac J Cancer Prev 2018; 19: 1319-24. doi: 10.22034/ APJCP.2018.19.5.1319

12. Vorgias G, lavazzo C, Savvopoulos P, Myriokefalitaki E, Katsoulis M, Kalinoglou $\mathrm{N}$, et al. Can the preoperative $\mathrm{Ca}-125$ level predict optimal cytoreduction in patients with advanced ovarian carcinoma? A single institution cohort study. Gynecol Oncol 2009; 112: 11-5. doi: 10.1016/j.ygyno.2008.09.020

13. Kang S, Kim TJ, Nam BH, Seo SS, Kim BG, Bae DS, et al. Preoperative serum CA-125 levels and risk of suboptimal cytoreduction in ovarian cancer: a meta-analysis. J Surg Oncol 2010; 101: 13-7. doi: 10.1002/jso.21398
14. Chi DS, Zivanovic O, Palayekar MJ, Eisenhauer EL, Abu-Rustum NR, Sonoda $Y$, et al. A contemporary analysis of the ability of preoperative serum CA-125 to predict primary cytoreductive outcome in patients with advanced ovarian, tubal and peritoneal carcinoma. Gynecol Oncol 2009; 112: 6-10. doi: 10.1016/j.ygyno.2008.10.010

15. Rodriguez N, Rauh-Hain JA, Shoni M, Berkowitz RS, Muto MG, Feltmate C, et al. Changes in serum CA-125 can predict optimal cytoreduction to no gross residual disease in patients with advanced stage ovarian cancer treated with neoadjuvant chemotherapy. Gynecol Oncol 2012; 125: 362-6. doi: 10.1016/j.ygyno.2012.02.006

16. Pelissier A, Bonneau C, Chéreau E, de La Motte Rouge T, Fourchotte V, Daraï $\mathrm{E}$, et al. CA125 kinetic parameters predict optimal cytoreduction in patients with advanced epithelial ovarian cancer treated with neoadjuvant chemotherapy. Gynecol Oncol 2014; 135: 542-6. doi: 10.1016/j.ygyno.2014.09.005

17. Wright AA, Bohlke K, Armstrong DK, Bookman MA, Cliby WA, Coleman RL, et al. Neoadjuvant chemotherapy for newly diagnosed, advanced ovarian cancer: Society of Gynecologic Oncology and American Society of Clinical Oncology clinical practice guideline. J Clin Oncol 2016; 34: 3460-73. doi: 10.1200/JCO.2016.68.6907

18. Orr B, Edwards RP. Diagnosis and treatment of ovarian cancer. Hematol Oncol Clin North Am 2018; 32: 943-64. doi: 10.1016/j.hoc.2018.07.010

19. Eisenhauer EA. Real-world evidence in the treatment of ovarian cancer. Ann Oncol 2017; 28(Suppl 8): viii61-5. doi: 10.1093/annonc/mdx443

20. Vergote I, Tropé CG, Amant F, Kristensen GB, Ehlen T, Johnson N, et al. Neoadjuvant chemotherapy or primary surgery in stage IIIC or IV ovarian cancer. N Engl J Med 2010; 363: 943-53. doi: 10.1056/NEJMoa0908806

21. Schwartz PE. Neoadjuvant chemotherapy for the management of ovarian cancer. Best Pract Res Clin Obstet Gynaecol 2002; 16: 585-96. doi: 10.1053/ beog.2002.0304

22. Morice P, Dubernard G, Rey A, Atallah D, Pautier P, Pomel C, et al. Results of interval debulking surgery compared with primary debulking surgery in advanced stage ovarian cancer. J Am Coll Surg 2003; 197: 955-63. doi: 10.1016/j.jamcollsurg.2003.06.004

23. Pecorelli S, Odicino F, Favalli G. Interval debulking surgery in advanced epithelial ovarian cancer. Best Pract Res Clin Obstet Gynaecol 2002; 16: 573-83. doi: 10.1053/beog.2002.0302

24. Zeng J, Yin J, Song X, Jin Y, Li Y, Pan L. Reduction of CA125 levels during neoadjuvant chemotherapy can predict cytoreduction to no visible residual disease in patients with advanced epithelial ovarian cancer, primary carcinoma of fallopian tube and peritoneal carcinoma. J Cancer 2016; 7: 2327-32. doi: 10.7150/jca.16761

25. Mahdi H, Maurer KA, Nutter B, Rose PG. The impact of percent reduction in CA-125 levels on prediction of the extent of interval cytoreduction and outcome in patients with advanced-stage cancer of Müllerian origin treated with neoadjuvant chemotherapy: Int J Gynecol Cancer 2015; 25: 823-9. doi: 10.1097/IGC.0000000000000434

26. Gupta M, Patel SM, Arora R, Tiwari R, Dave P, Desai A, et al. Does preoperative CA-125 cutoff value and percent reduction in CA-125 levels correlate with surgical and survival outcome after neoadjuvant chemotherapy in patients with advanced-stage ovarian cancer? - Our experience from a tertiary cancer institute. South Asian J Cancer 2020; 9: 30. doi: 10.4103/sajc. sajc_53_17

27. Kessous R, Wissing MD, Piedimonte S, Abitbol J, Kogan L, Laskov I, et al. CA-125 reduction during neoadjuvant chemotherapy is associated with success of cytoreductive surgery and outcome of patients with advanced high-grade ovarian cancer. Acta Obstet Gynecol Scand 2020; 99: 933-40. doi 10.1111/aogs.13814

28. Mueller JJ, Zhou QC, lasonos A, O'Cearbhaill RE, Alvi FA, El Haraki A, et al. Neoadjuvant chemotherapy and primary debulking surgery utilization for advanced-stage ovarian cancer at a comprehensive cancer center. Gynecol Oncol 2016; 140: 436-42. doi: 10.1016/j.ygyno.2016.01.008

29. Kehoe S, Hook J, Nankivell M, Jayson GC, Kitchener H, Lopes T, et al. Primary chemotherapy versus primary surgery for newly diagnosed advanced ovarian cancer (CHORUS): an open-label, randomised, controlled, non-inferiority trial. Lancet 2015; 386: 249-57. doi: 10.1016/S0140-6736(14)62223-6

30. Mahner S, Trillsch F, Chi D, Harter P, Pfisterer J, Hilpert F, et al. Neoadjuvant chemotherapy in ovarian cancer revisited. Ann Oncol 2016; 27(Suppl 1): i30-2. doi: 10.1093/annonc/mdw092

31. Machida H, Tokunaga H, Matsuo K, Matsumura N, Kobayashi Y, Tabata T, et al. Survival outcome and perioperative complication related to neoadjuvant chemotherapy with carboplatin and paclitaxel for advanced ovarian cancer: a systematic review and meta-analysis. Eur J Surg Oncol 2020; 46: 868-75. doi: 10.1016/j.ejso.2019.11.520

32. du Bois A, Baert T, Vergote I. Role of neoadjuvant chemotherapy in advanced epithelial ovarian cancer. J Clin Oncol 2019; 37: 2398-405. doi: 10.1200/JCO.19.00022 